\title{
Modeling of Radial Flow on a Non-Contact End Effector for Robotic Handling of Non-Rigid Material
}

\author{
E. Toklu', F. Erzincanli \\ Duzce University, Duzce, Turkey \\ *ethemtoklu@duzce.edu.tr
}

\begin{abstract}
In this study a numerical model on radial flow and pressure distribution showing regions of negative values which tend to levitate products is developed. The end effector operates on the principle of generating a high-speed fluid flow between the end effector and product surface thereby creating a vacuum which levitates the product. The NavierStokes equations and the equation of continuity describing the flow between the nozzle and material are numerically solved by finite volume discretization method. The lifting forces and conditions are discussed by comparing numerical results with experimental findings.
\end{abstract}

Keywords: Radial flow, robotic handling, non-contact end effector, levitation, lifting force.

\section{Introduction}

Available systems for the handling and packaging of discrete food products, both old and new, rely heavily on the role of the human operator. The human provides a high degree of dexterity and flexibility which can be readily applied to a wide variety of food products ranging from fish fillets, jelly blocks, meat and poultry portions, to sliced ham and other sliced processed meats. Each of these products features variations in texture, color, shape and size. The food products are also often delicate, easily marked or bruised, compliant, adhesive and slippery. Conventional handling and gripping technology which features two or more jaws is not directly applicable. All forms of contact end effectors can potentially cause product bruising and deformation. The air permeability, elasticity, surface adhesiveness and smoothness, shapes and weight of food products, as mechanical properties, are their most important characteristics from the point of view of handling and invariably have to be considered when selecting end effectors to handle food products.

The end part of the robot or programmable machine arm is referred to as an "end effector"; thus, the end effector is the device mounted at the distal end of a robot arm, enabling it to pick up an object and hold, manipulate, transfer, place and release it accurately in a discrete position $[7,11]$.
Hence, the gripper is the all-important mechanical interface between the robot and its environment without which, in many circumstances, the robot cannot function effectively, irrespective of the degree of sophistication it may otherwise possess.

All currently available methods for gripping a workpiece fall under one of two general categories: clamping and attracting methods, by Tella et al. [13]. The first category, clamping methods, is comprised of jaw-type devices which exert pressing forces on at least two opposing elements of a workpiece. The workpiece is held in place by the resulting frictional forces. The second category, attracting methods, is comprised of vacuum, magnetic, adhesive, and all other methods characterized by attractive holding forces. There are two major advantages of clamping end effectors. First of all, these end effectors are less dependent than attracting methods on workpiece surface conditions and irregularities. Secondly, their gripping force is only limited by their clamping force. The major disadvantage of clamping methods is that they require access to more than one surface of the workpiece for gripping to occur. The clamping end effectors are intended for handling rigid, usually three-dimensional, objects. Despite the clamping end effectors being successfully used in manufacturing, it is difficult to use them for handling non-rigid materials such as jelly blocks and sliced meats [1-2]. 
Vacuum end effectors on the other hand require only single surface access to the workpiece for successful gripping. The gripping force of vacuum methods is limited by the accessible surface area of the workpiece holding side. Suction end effectors can also remove essential fluids and other particles from the product which can result in contaminated pipelines. Ideally, there needs to be a non-contact handling device which can be simply controlled in much the same way as a suction end effector and which can introduce a temporary level of rigidity into the product in order to facilitate high speed handling and manipulation $[5,9,12]$. Several investigators have studied radial flow on thrust bearings, aerosol impactors, vertical take-off and landing vehicles, and electro-erosion machining. Ferreira et al. [4] investigated the pressure distribution in laminar radial flow through parallel concentric disks. With increasing mass flow, the Reynolds number at the inlet may exceed a critical value, so that turbulent flow will exist for some distance downstream of the inlet corner. This type of flow causes the attracting force between the two disks. When the velocity, which decreases with increasing radius, has fallen sufficiently for the local Reynolds number to become sub-critical, a reverse transition from turbulent to laminar flow results. If the mass flow is increased still further, the flow in the channel becomes fully turbulent, and eventually the inertia terms predominate over the viscous terms in determining the pressure distribution. As the radius increases, the pressure raises in the radial direction $[1,8]$; therefore, the attracting forces decrease. Possamai et al. [10] performed a numerical and an experimental investigation of the incompressible laminar and isothermal flow in a radial diffuser having one disk inclined with respect to the other. For some combinations of Reynolds number, gap distance and inclination between disks, the pressure distribution showed regions of negative values

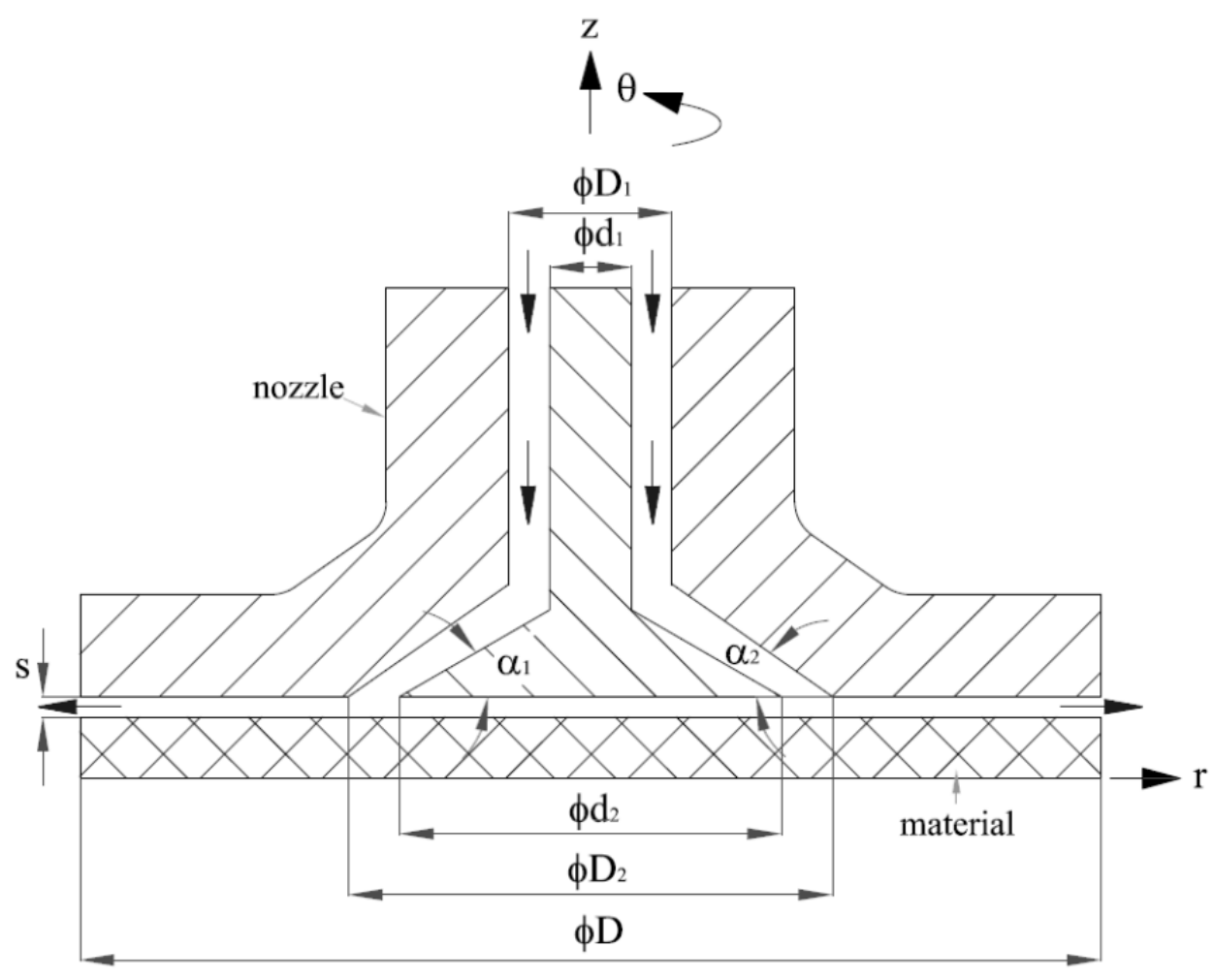

Figure 1. Geometry of nozzle with axial feeding and material. 
which tended to pull the frontal disk towards the valve seat and also produce a restoring moment tending to make the disks parallel.

The aim of this research was to develop a numerical model which explained the radial flow phenomena from the fluid mechanics point of view in the developed non-contact end effector that could handle non-rigid, relatively flat and thin food products with slippery or sticky surfaces, particularly jelly blocks and sliced meat, meeting the rigorous hygienic standards of the food industry and to carry wool non-contact for textile industry. Sliced meat and jelly blocks are quite good examples of delicate food products from the handling point of view as they are very flexible, covered with either slippery or sticky substances, relatively flat and thin. The thickness dimension of such products is much smaller than their surface area dimensions $[3,6]$. In this way, an efficient and effective design strategy is proposed for a noncontact effector. In this strategy, a numerical model is developed to calculate radial flow

\section{Experimental setup}

The experimental setup was designed and assembled by Erzincanli [3] as flexible as possible in order to carry out different experiments. The end effectors with different nozzle head configurations can easily be replaced with different ones using the screwed rods and nuts. The compressed air supply tube from the air tank can be connected to a replacement end effector system with a flexible connection, reducing the time to change over.

The experimental setup consists of several pieces of basic and auxiliary equipment. A schematic representation of the equipment that forms the experimental rig is illustrated in Figure 2.

Compressed air from a source enters through a pipe at the center of the upper disk and, after striking the lower disk (Figure 2), flows radially outwards between the two disks which are circular. The system is called a "radial flow nozzle" and consists of two narrowly spaced circular disks placed parallel to each other and perpendicular to a central inlet pipe. The radial outflow of air between the parallel disks causes either an attracting or a repelling force to exist between the disks. In general, in order to create an attracting force, the clearance gap of a nozzle must be very small as compared with the diameter of the central tube and the radial distance through which the fluid flows.

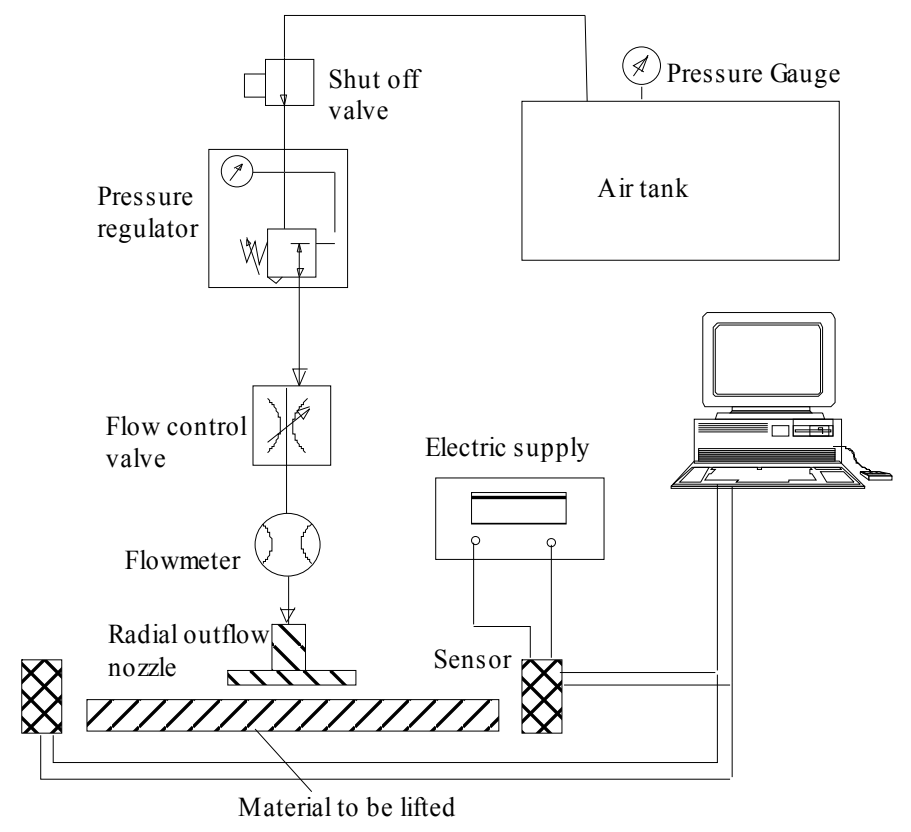

Figure 2. A schematic representation of the experimental rig. 
By experimentation, the jelly block is lifted up by vacuum forces which is shown in Figure 3. This was first discovered by Erzincanli [3]. In this study, a model is developed to explain it mathematically. $d_{1}, D_{1}, d_{2}, D_{2}$ are the diameters and $\alpha_{1}, \alpha_{2}$ are the angles of the nozzles as shown in Figure 1. These parameters were considered as $d_{1}=3 \mathrm{~mm}, D_{1}=5$ $\mathrm{mm}, \mathrm{d}_{2}=8 \mathrm{~mm}, \mathrm{D}_{2}=9 \mathrm{~mm}, \mathrm{~s}=0,2 \mathrm{~mm}, \mathrm{a}_{1}=30^{\circ}$, $\alpha_{2}=35^{\circ}$.

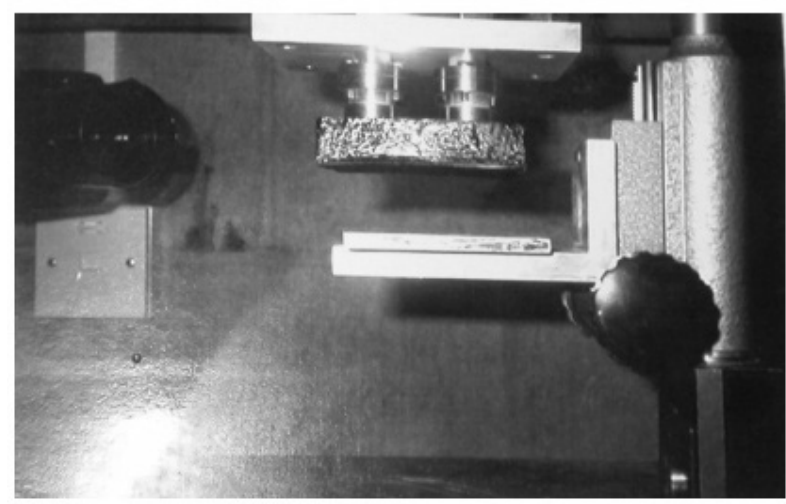

Figure 3. The jelly block during the lifting process.

\section{Mathematical formulation of problem}

The governing equations of the problem will be obtained according to Figure 1 . The basic assumption is incompressible steady flow for a Newtonian fluid. When the flow is axisymmetrical, the Navier-Stokes and continuity equations governing the flow are represented in cylindrical coordinates as follows:

Continuity equation:

$$
\frac{\partial}{\partial \theta}(\rho \mathrm{u})+\frac{\partial}{\partial \mathrm{r}}(\rho \mathrm{rv})+\frac{1}{\mathrm{~s}} \frac{\partial}{\partial \mathrm{z}}(\rho \mathrm{rw})=0
$$

Momentum (Navier-Stokes) equations in the $r$ direction;

$$
\begin{gathered}
\frac{1}{r}\left[\frac{\partial}{\partial \theta}(\rho u v)+\frac{\partial}{\partial r}\left(\rho r v^{2}\right)+\frac{1}{s} \frac{\partial}{\partial z}(\rho r w v)\right]=-\frac{\partial p}{\partial r}+ \\
\frac{\mu}{r}\left[\frac{\partial}{\partial \theta}\left(\frac{1}{r} \frac{\partial v}{\partial \theta}\right)+\frac{\partial}{\partial r}\left(r \frac{\partial v}{\partial r}\right)+\frac{1}{s} \frac{\partial}{\partial z}\left(\frac{r}{s} \frac{\partial v}{\partial z}\right)\right]-\frac{2 \mu}{r^{2}} \frac{\partial u}{\partial \theta}-\frac{\mu v}{r^{2}}+\frac{\rho u^{2}}{r}
\end{gathered}
$$

Momentum (Navier-Stokes) equations in the $\theta$ direction;

$$
\begin{array}{r}
\frac{1}{r}\left[\frac{\partial}{\partial \theta}\left(\rho u^{2}\right)+\frac{\partial}{\partial r}(\rho r v u)+\frac{1}{s} \frac{\partial}{\partial z}(\rho r w u)\right]=-\frac{1}{r} \frac{\partial p}{\partial \theta}+ \\
\frac{\mu}{r}\left[\frac{\partial}{\partial \theta}\left(\frac{1}{r} \frac{\partial u}{\partial \theta}\right)+\frac{\partial}{\partial r}\left(r \frac{\partial u}{\partial r}\right)+\frac{1}{s} \frac{\partial}{\partial z}\left(\frac{r}{s} \frac{\partial u}{\partial z}\right)\right]+\frac{2 \mu}{r^{2}} \frac{\partial v}{\partial \theta}-\frac{\mu u}{r^{2}}-\frac{\rho u v}{r}
\end{array}
$$

Momentum (Navier-Stokes) equations in the $z$ direction;

$$
\begin{gathered}
\frac{1}{r}\left[\frac{\partial}{\partial \theta}(\rho u w)+\frac{\partial}{\partial r}(\rho r v w)+\frac{1}{s} \frac{\partial}{\partial z}\left(\rho r w^{2}\right)\right]=-\frac{1}{s} \frac{\partial p}{\partial z}+ \\
\frac{\mu}{r}\left[\frac{\partial}{\partial \theta}\left(\frac{1}{r} \frac{\partial w}{\partial \theta}\right)+\frac{\partial}{\partial r}\left(r \frac{\partial w}{\partial r}\right)+\frac{1}{s} \frac{\partial}{\partial z}\left(\frac{r}{s} \frac{\partial w}{\partial z}\right)\right]
\end{gathered}
$$

Reynolds number:

$$
R e=\frac{\left(D_{1}-d_{1}\right) w}{\vartheta}
$$

Boundary conditions are defined as solid walls, inflow and outflow for equations (1) - (4). The nonslip and impermeable boundary conditions are set at the solid wall and $u=v=w=0$. A parabolic boundary condition is imposed at the outflow and $w=\partial u / \partial r=\partial(r v) / \partial r=0$. For the Reynolds number considered here, separation always occurred as the fluid turned from the feeding orifice to the material (Figure 1).

\section{Numerical solution}

A finite volume discretization scheme was applied to solve the governing differential equations. According to this practice, the solution domain is divided into small non-overlapping control volumes and the continuity and momentum differential equations are integrated over each control volume. The resulting system of algebraic equations is solved using a combination of the Thomas algorithm and the Gauss-Seidel method. Prior to solving the algebraic equations, the discretized form of the continuity equation was transformed into an equation for pressure using the SIMPLE methodology [10].

A key issue in the accuracy of the numerical solution is the interpolation scheme employed to evaluate the variables at the control volume faces. The QUICK scheme was adopted, yielding a second-order accuracy for the interpolated values. 
In some cases, numerical instabilities introduced by the QUICK methodology precluded convergence. Even though a consistent QUICK scheme was applied here, those instabilities were only eliminated when the power-law scheme was employed in the axial and circumferential directions. As observed from several numerical experiments performed, the radial direction is the most critical for numerical accuracy of the solution, and the second-order interpolation scheme was maintained for that direction.

Due to the strong non-linearity of the equations, under-relaxation coefficients were required. For the velocity components, these coefficients were 0.2 and for pressure 0.4. Convergence was stopped when the maximum residual of the algebraic equations was less than $10^{-6}$; at the beginning of the computations the value of the maximum residual was $10^{-1}$. All the results to be presented were obtained with a mesh consisting of fine grid points. The final mesh adopted in performing the computations was chosen after conducting several grid independence tests.

\section{Results and discussions}

The main focus of the present study is compatibility of numerical results with experimental finding. In this regard, the flow through the nozzle in Figure 1 was investigated for one displacement and five Reynolds number $(\operatorname{Re}=250,750,1250$, 1750 and 2250). Qualitative agreements of the numerical model were evaluated first through comparisons with experimental results.

Results for the pressure and velocity distribution along the nozzle are presented in figure 4, 5, 6, 7 and
8 for different Reynolds number. The jelly with standard ingredients was molded with standard dimensions and four different thicknesses to obtain weights of $35,70,105$ and 140 grams. The results of the experimentation for the weight of 70 grams are illustrated in figure 3 that shows a jelly block during the lifting process. It can be concluded that the clearance gap increases with increasing volumetric air flow rate with experimental and finite volume model. The clearance gap between the jelly block and the nozzle could not be shown because of inadequate focusing of the figure.

The pressure and velocity distribution profile along the nozzle shows similar behaviors for the different Reynolds number. The occurrence of levitation force, which lifts the material up, depends on the negative pressure between the nozzle body and material. According to the numerical results, it can be seen that increasing the Reynolds number increases the negative pressure. That means that a high negative pressure leads to more lifting force.

In the experimental study, four nozzles were configured and used to lift various materials during experimentation. The surface area of the standard specimen was divided equally into four sections. Nozzle heads were positioned over the center of each divided section; thus, the forces were evenly distributed across the specimen surface. The distance between the nozzle heads, from edge to edge, was 11 millimeters. The total surface area of the four nozzles covered $36.9 \%$ of the total area of the specimen's top surface. In order to supply air to this configuration, the air distributor for four nozzles was used.

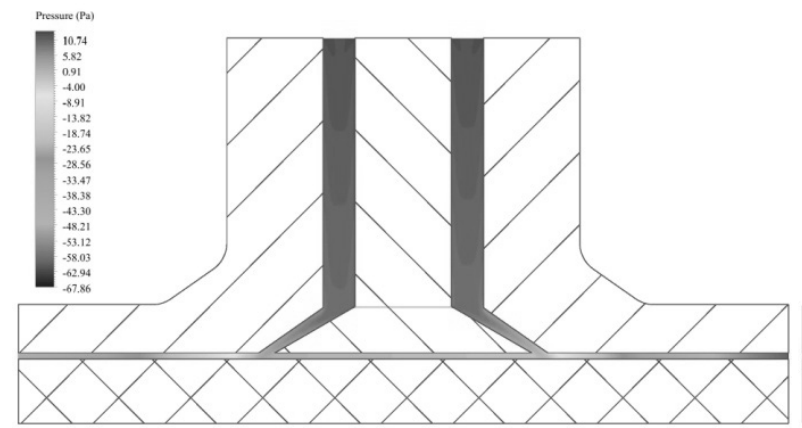

(a)

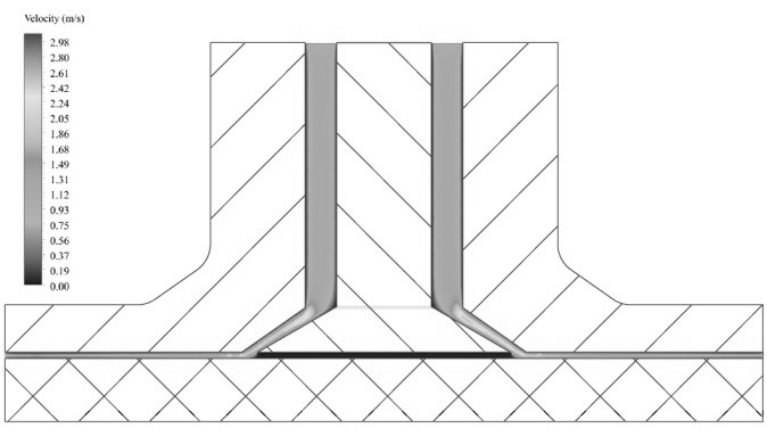

(b)

Figure 4. Numerical results for (a) pressure (b) velocity distribution along the nozzle for $\operatorname{Re}=250$. 


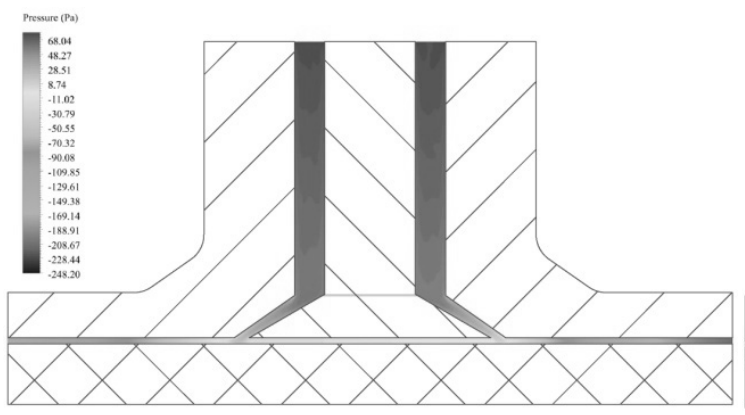

(a)

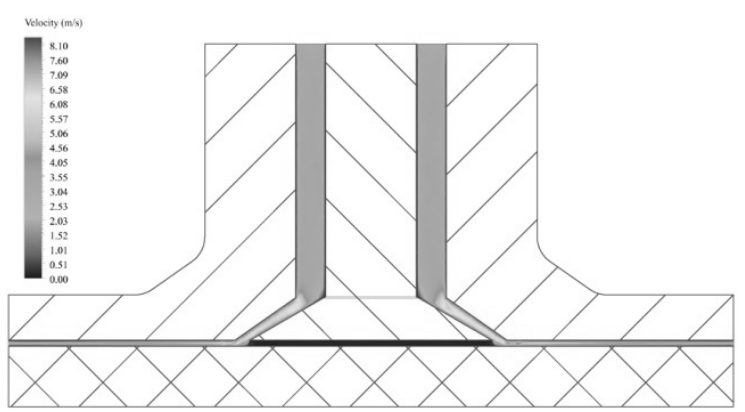

(b)

Figure 5. Numerical results for (a) pressure (b) velocity distribution along the nozzle for $\mathrm{Re}=750$.

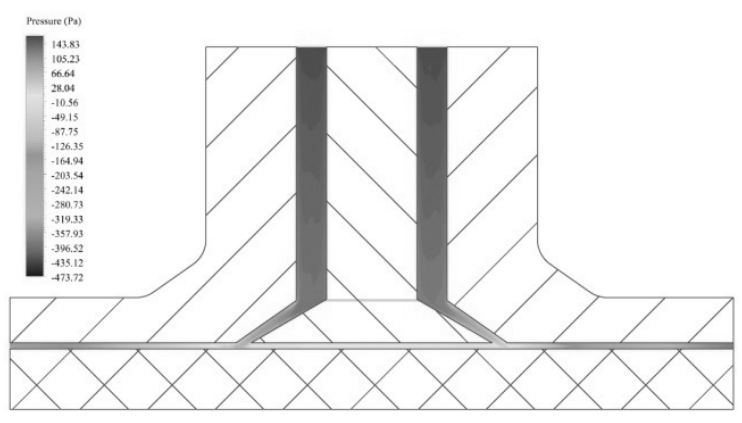

(a)

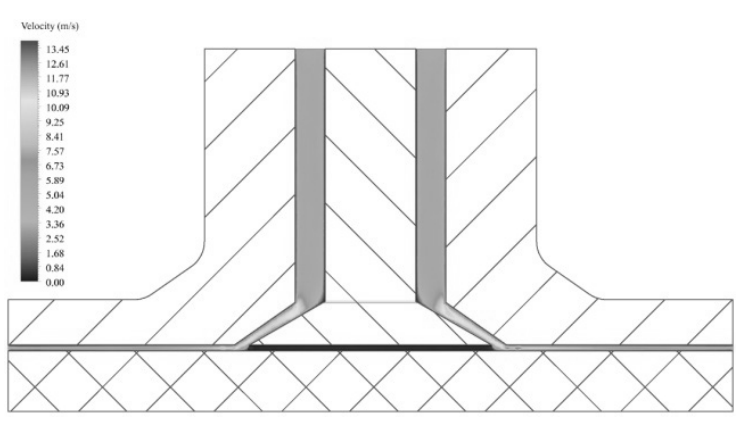

(b)

Figure 6. Numerical results for (a) pressure (b) velocity distribution along the nozzle for $\operatorname{Re}=1250$.

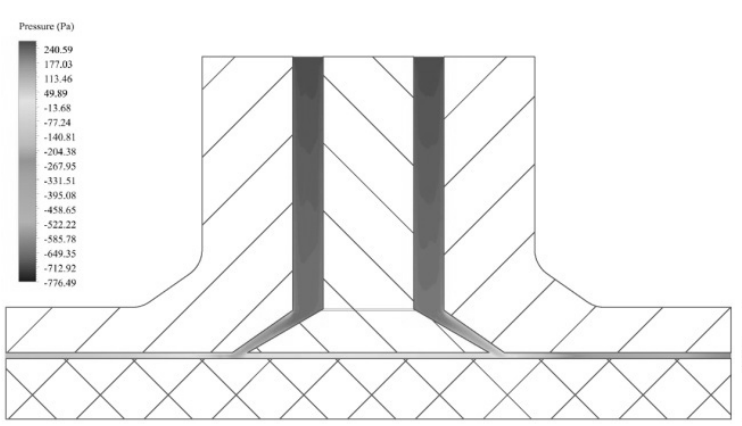

(a)

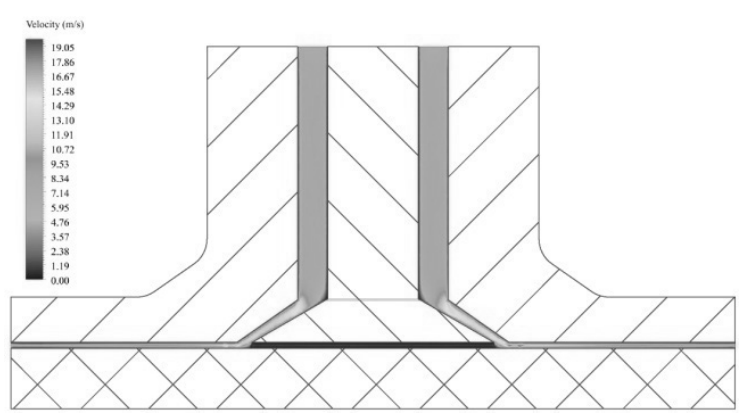

(b)

Figure 7. Numerical results for (a) pressure (b) velocity distribution along the nozzle for $\operatorname{Re}=1750$. 


\section{Conclusions}

The main goal of this study was the development of a numerical model which had qualitative agreement with the experimental finding. A very good agreement obtained between computation and experimental results validated both the numerical methodology and the experiments. From the experimental and numerical results, it was found that the pressure and velocity profile is very sensitive to the nozzle design parameters. Higher Reynolds number leads to higher vacuum force, therefore, heavier materials can be levitated.

A numerical model for the radial flow on a noncontact end effector was outlined in detail regarding the experimental results. An operational sequence and a conceptual application of the noncontact end effector in a practical handling environment were introduced. At the end, the experimental results show that a nozzle can be designed and studied in a computer environment before it is manufactured. This will save time in the design process and prevent product loss.

It was successfully achieved by developing a range of non-contact end effectors and by applying them to handle regularly-shaped rigid and non-rigid materials with relatively smooth surfaces both numerically and experimentally. It was found that these end effectors can also lift materials with porous structures and surfaces covered by viscous substances. This research is unique in being able to handle non-rigid materials using the radial outflow phenomena. One of the difficulties encountered during this research was the lack of relevant theoretical and practical information; therefore, this research may be regarded as an initial investigation in this field.

\section{References}

[1] Erzincanli F and Sharp JM, "Non-contact end effector for robotic handling of compliant products", Proceedings of the Thirty-first Inter. MATADOR Conf., Manchester, 629-634, 1995.

[2] Erzincanli F, Sharp JM and Dore AM, "Grippers for handling of non-rigid food products", Proceeding of EURISCON ‘94, Malaga. 3, 798-806, 1994.

[3] Erzincanli F, "A non-contact end effector for robotic handling of non-rigid materials", PhD Thesis, University of Salford, Salford, 1995.

[4] Ferreira RTS, Deschamps CJ and Prata AT, "Pressure distribution along valve reeds of hermetic compressors", Experimental Thermal and Fluid Sciences, 2, 201-207, 1989.

[5] Heilala J, Ropponen T and Airila M, "Mechatronic design for industrial grippers", Mechatronics, 2/3, 239255, 1992.

[6] Kayabasi O, Erzincanli F, "Shape optimization teeth profile of flexspline for harmonic drive by finite element method", Materials and Design, volume 28, issue 2, Pages 441-447, 2007.

[7] Madwed A, "A new gripper system to handle many different parts for assembly by robots", Robotics International of SME Robots Conference, 11.83-11.96, (1985).

[8] Moller PS. "Radial flow without swirl between parallel discs", The Aeronautical Quarterly, May, pp: 163-186, 1963.

[9] Pham D T and Heginbotham WB, "Robot Grippers", IFS Bedford, and Springer-Verlag Berlin, 1986.

[10] Possamai FC, Ferreira RTS and Prata AT, "Pressure distribution in laminar radial flow through inclined disks", Int. J. of Heat and Fluid Flow, 22, 440449, 2001.

[11] Schneider RT, "End effectors take hold-in robotics", Hyd. and Pneums., 40-42, 1992.

[12] Simonton W, "Robotic end effector for handling greenhouse plant material", Transaction of the ASAE, 34/6, 2615-2621, 1991.

[13] Tella R, Birk JR and Kelley RB, "General purpose hands for bin-picking robots", IEEE Transaction on System, Man and Cybernetics, 12/6, 828-837, 1982. 
